\title{
Propuesta de una plataforma de bajo costo basada en Internet de las Cosas para Agricultura Inteligente
}

\author{
Propuesta de una Plataforma de Bajo Costo \\ Basada en Internet de las Cosas para Agricultura \\ Inteligente
}

Nuñez-Agurto Daniel Universidad de las Fuerzas Armadas ESPE adnunez1@espe.edu.ec

Benavides-Astudillo Eduardo Escuela Politécnica Nacional Universidad de las Fuerzas Armadas-ESPE diego.benavides@epn.edu.ec / debenavides@espe.edu.ec

Rodríguez German Escuela Politécnica Nacional Universidad de las Fuerzas Armadas-ESPE german.rodriguez@epn.edu.ec / gerodriguez10@espe.edu.ec

Salazar Diego

Universidad de las Fuerzas Armadas-ESPE drsalazar@espe.edu.ec

Revista Cumbres Vol.6 №1

Versión electrónica ISSN 1390-3365 http://investigacion.utmachala.edu.ec/revistas/index.php/Cumbres 


\title{
RESUMEN
}

La agricultura tradicional se ve afectada en rendimiento y calidad por diferentes factores ambientales externos. Para enfrentar este problema se vuelve necesaria la innovación tecnológica, permitiendo de esta manera, optimizar recursos invertidos en los cultivos para volverlos más productivos y rentables. Una de las alternativas de innovación para el sector agrícola es la implementación de Internet de las Cosas (IoT). Es así que este trabajo propone la implementación de una solución de hardware y software de bajo costo, que permita almacenar y analizar datos ambientales de los cultivos de manera centralizada y remota, para posteriormente realizar pronósticos con mayor precisión en indicadores ambientales como la humedad y la temperatura. El desarrollo de esta propuesta utilizó microcontroladores NodeMCU ESP8266, sensores de temperatura/humedad AM2302, sensores de lluvia MH-RD, y se desarrolló un dashboard con el fin de analizar los datos en tiempo real utilizando la herramienta node-red. Para que el sistema interactúe con los sensores remotos, se usó una conexión inalámbrica wifi por medio del protocolo MQTT. Las pruebas de esta propuesta se las realizaron en las parcelas agrícolas de la Universidad de las Fuerzas Armadas ESPE Sede Santo Domingo. Los resultados obtenidos mediante la operación del prototipo tuvieron mayor precisión en comparación de un termohigrómetro y la aplicación google weather.

Palabras clave: Agricultura inteligente, Internet de las Cosas, Node-Red, Micropython.

\begin{abstract}
Traditional agriculture is affected in yield and quality by different external environmental factors. To face this problem, technological innovation becomes necessary since it allows the optimization of resources invested in the crops to make them more productive and profitable. One innovation alternative for the agricultural sector is the implementation of the Internet of Things (IoT). Thus, this work proposes the implementation of a low-cost hardware and software solution that allows to store and analyze environmental data of the crops in a centralized and remote way. This data will help to later make forecasts with greater precision in environmental indicators such as humidity and temperature. For the development of this proposal, NodeMCU ESP8266 microcontrollers, DTH22 temperature / humidity sensors, and MH$\mathrm{RD}$ rain sensors were used. A dashboard was developed to analyze the data in real time by using the node-red tool. In order for the system to interact with the remote sensors, a wireless connection was used through the MQTT
\end{abstract}

\section{Cumbres}


protocol. The trials of this proposal were made in the agricultural parcels of the Universidad de las Fuerzas Armadas ESPE, Santo Domingo Headquarters. The results obtained through the operation of the prototype had greater precision compared to a thermo-hygrometer and the Google weather application.

Keywords: Smart Agriculture, Internet of Things, Node-Red, Micropython.

\section{INTRODUCCIÓN}

Internet de las Cosas (IoT) es una tecnología que esta está tomando fuerza en distintos campos como en: las ciudades, transporte, agricultura, energía, y edificios inteligentes, inclusive existe un despliegue de medidores inteligentes conectados directamente a la red eléctrica. Esta tecnología tiene como objetivo mejorar la calidad de vida de las personas. Los dispositivos más utilizados por IoT son los microcontroladores y sensores que permiten la interconexión y envió de datos, utilizando distintos tipos de protocolos (Zanella, Bui, Castellani, Vangelista, \& Zorzi, 2014),(Siano, 2014), también posibilitan recibir y ejecutar comandos, mediante aplicaciones específicas instaladas en un computador, o alojadas en la nube, e incluso existen aplicaciones desarrolladas para dispositivos móviles. Los comandos que se envían pueden ser: encender las luces de una casa, abrir el garaje o encender el aire acondicionado.

Para el año 2020 se estima generar unos 2,3 Zetabytes de trafico IP (Internet Protocol) Global, lo que representará un crecimiento de 72.5 Exabytes mensuales. Por la creciente demanda de conexiones a Internet, se calculan unos 26300 millones de dispositivos conectados a Internet en ese año; representando el 71\% del tráfico generado por los dispositivos que no son computadores (CISCO, 2018). La enorme cantidad de datos que generará IoT a partir de diferentes dispositivos se debe poder analizar, comprender y sobre todo generar conocimiento (Barnaghi, Sheth, \& Henson, 2013). El análisis de datos de IoT puede centrarse en aplicaciones que busquen predecir la temperatura y humedad en zonas agrícolas, logrando optimizar el consumo de energía que utilizan las bombas de riego en los cultivos o ventiladores en granjas avícolas (Montoya, Colorado, Muñoz, \& Golondrino, 2017). Este concepto de interacción con dispositivos físicos permitirá aplicar nuevos métodos y técnicas sobre plataformas centralizadas, que permitan un análisis inteligente de la información en tiempo real.

El crecimiento de la población mundial está generando gran preocupación por la demanda de alimentos, mientras el calentamiento global continúa afectando las diferentes áreas del sector agrícola (Zhang, Peng \& Zhang, Junjie \& Chen, 2016). Además, se debe considerar que países subdesarrollados realizan el proceso de cultivo con base en el esfuerzo humano, reduciendo sus beneficios, a diferencia de los países desarrollados en los que se encuentran automatizados la mayor parte de sus cultivos, mejorando sustancialmente sus ganancias (Ma, Zhou, Li, \& Li, 2011). La implementación de 
dispositivos IoT en la agricultura ayudará a optimizar los recursos invertidos en los cultivos, haciéndolos más rentables y productivos. Estos dispositivos, en la actualidad pueden ser accesibles para los agricultores debido a su bajo costo (Heble et al., 2018). Este flujo de datos en tiempo real permitiría desarrollar sistemas inteligentes que tomen decisiones, orientados a optimizar la producción y que sean más competitivos en mercados internacionales (Viani, Bertolli, Salucci, \& Polo, 2017). Inclusive los agricultores se deberían organizar para integrar plataformas de análisis de datos, que sería la parte que demandaría más inversión y soporte especializado.

El presente trabajo propone implementar una plataforma de monitoreo y un dispositivo de medición de variables climáticas de bajo costo, basado en IoT, para la monitorización agrícola. Esta solución fue desarrollada con herramientas de código abierto y hardware. El resto del documento está organizado de la siguiente manera: La sección 2 se enfoca en mostrar los materiales y métodos utilizados, la sección 3 analiza los resultados obtenidos así como la comparación con un dispositivo de precisión común, y finalmente en la sección 4 se exponen las conclusiones del trabajo y trabajos futuros propuestos.

\section{Trabajos relacionados}

En la siguiente sección se presenta las plataformas relacionadas con IoT, que se propusieron en la literatura.

\section{A. Principales arquitecturas de IoT}

Los autores (Jayaraman, Palmer, Zaslavsky, \& Georgakopoulos, 2015) integran la plataforma Open IoT recientemente desarrollada y que es aplicable en varios casos de uso con la Agricultura Digital (Phenonet) para desarrollar una ontología agrícola semánticamente mejorada. En el trabajo de (Qiu, Xiao, \& Zhou, 2013) se muestra una plataforma de monitoreo inteligente y una estructura de sistema para el ecosistema de instalaciones agrícolas basado en IOT. De manera similar (Guo \& Zhong, 2015) presenta una nueva estructura integrada de un sistema de adquisición de datos y el sistema de control inteligente de las instalaciones agrícolas. En (Khattab, Abdelgawad, \& Yelmarthi, 2017) se expone una arquitectura IoT personalizada para aplicaciones agrícolas de precisión, con una arquitectura de tres capas, que recopila los datos necesarios y los transmite a un back-end basado en la nube donde la información es procesa y analizada.

\section{B. Estaciones de monitoreo basadas en IoT}

El trabajo de los autores (Munandar et al., 2018) muestra la implementación del sistema meteorológico basado en la comunicación en serial. Posteriormente, las investigaciones de (Halder \& Sivakumar, 2018) integran la opción de conectividad a Internet utilizando módulos GSM y Ethernet, en ambos casos, los parámetros monitoreados fueron humedad, temperatura, lluvia, 
radiación solar y radiación UV. En los estudios de (R. K. Math \& Dharwadkar, 2017; Saini, Thakur, Ahuja, Sabharwal, \& Kumar, 2016) utilizaron en sus aplicaciones el protocolo Zigbee, lograron medir datos meteorológicos, incluida la presión barométrica, la temperatura del punto de rocío, la temperatura del aire, la velocidad y la dirección del viento, la humedad relativa.

En el trabajo de (Mangundu, Mateus, Zodi, \& Johson, 2018) se desarrolló un sistema de monitoreo de lluvia utilizando redes de sensores inalámbricos, las mediciones son enviadas a un servidor web público a través del Servicio General de Radio por Paquetes (GPRS). (Shaout, Li, Zhou, \& Awad, 2015) presenta un diseño integrado de una estación meteorológica de bajo costo, con la medición de tres parámetros climáticos; velocidad del viento, dirección del viento y la temperatura. Los parámetros medidos se utilizan para medir la temperatura del viento y el índice de apósito mediante el cálculo y un sistema inteligente incorporado. Mientras que (R. K. M. Math \& Dharwadkar, 2019) propone una estación meteorológica local en tiempo real basada en IoT para la Agricultura de Precisión que proporciona a los agricultores un medio de automatizar sus prácticas agrícolas (riego, fertilización, cosecha) en el momento adecuado. El sistema permite a los agricultores realizar las tareas agrícolas en tiempo real, lo que a su vez les ayuda a utilizar los recursos agrícolas de manera eficiente y en el momento en que los cultivos lo necesitan.

El sistema propuesto por (Nageswara Rao \& Sridhar, 2018) fue desarrollado a partir de la información enviada por los sensores, estimando la cantidad de agua necesaria. Para este proceso utilizan dos sensores para llevar los datos a la estación base, la temperatura del suelo, la humedad y la duración de la luz solar por día. Por su parte (Dholu \& Ghodinde, 2018) propone el desarrollo de un nodo sensor capaz de medir parámetros agrícolas como la humedad, la temperatura y la humedad relativa alrededor de la planta, la intensidad de la luz y con esta información es capaz de generar una señal de actuación. En esta propuesta, los nodos de los sensores también son capaces de enviar los datos a la nube. En el trabajo de (Mat, Kassim, \& Harun, 2014) se muestran los requisitos de diseño, la arquitectura del sistema, la implementación, los resultados de las pruebas del sistema de WSN para Agricultura de Precisión en un invernadero, el software monitorea los datos de los sensores en un circuito de retroalimentación que activa los dispositivos de control según el valor del umbral.

\section{MATERIALES Y MÉTODOS}

En esta sección se presenta el diseño del presente estudio, que comprende la arquitectura del dispositivo electrónico, sus características, plataforma de monitoreo y el proceso de desarrollo. En la Fig. 1 podemos observar cómo está diseñada la implementación de esta investigación. 
Figura 1. Esquema general de la implementación del dispositivo IoT

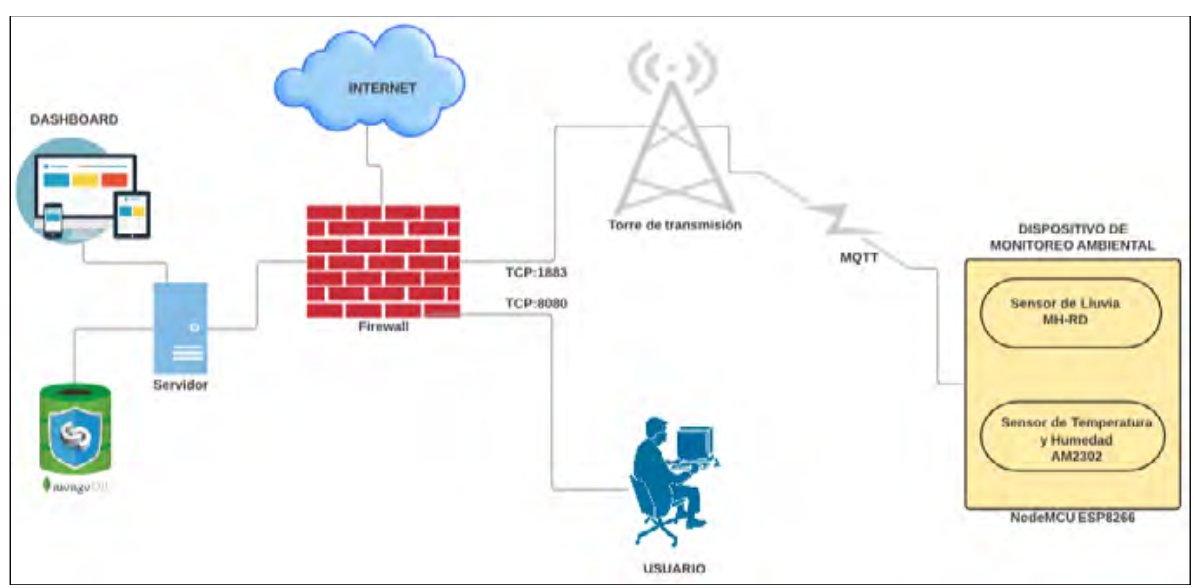

\section{Dispositivo de medición ambiental}

\section{Funcionamiento del dispositivo}

El dispositivo desarrollado utiliza un microcontrolador nodeMCU ESP8266 que integra un kit de desarrollo y un firmware de código abierto, permitiendo desarrollar productos para IoT (Saputra \& Lukito, 2017); un sensor AM2302(DTH22), para obtener datos humedad y temperatura con alta precisión a de bajo que costo, el cual emite una señal digital a través de un pin de datos (Kodali \& Mahesh, 2016); un sensor de lluvia MH-RD capaz de detectar la presencia de lluvia al momento que las gotas caen sobre la placa del sensor (Openhacks, 2016). La función principal del dispositivo es capturar información ambiental de humedad, temperatura y de lluvia, que posteriormente será enviada a un servidor de mensajes o bróker, para que sea procesada y analizada, en la Fig. 2 podemos observar el algoritmo que permite hacer este proceso.

El experimento utiliza el protocolo MQTT(Message Queue Telemetry Transport) para el envío de información, debido a que este protocolo es usado para la comunicación M2M (Machine to Machine) en IoT. Así, su principal característica es que permite la comunicación entre sensores, utilizando poco ancho de banda y además funciona en redes inalámbricas con alta latencia (Kodali \& Soratkal, 2017). El proceso de captura de las variables ambientales es realizado mediante una librería integrada en el dispositivo nodeMCU, en el lenguaje de programación Mycropython, por medio de un programa desarrollado para este propósito. Luego, usando el programa implementado, los datos son enviados periódicamente cada cinco minutos al servidor que se encuentra en el centro de datos de la Universidad de las Fuerzas Armadas ESPE, utilizando el protocolo MQTT y a través de una conexión inalámbrica wifi, establecida entre el nodeMCU y la torre de transmisión en la banda de $2.4 \mathrm{GHz}$. 
Figura 2. Algoritmo para captura y enviar datos de un sensor.

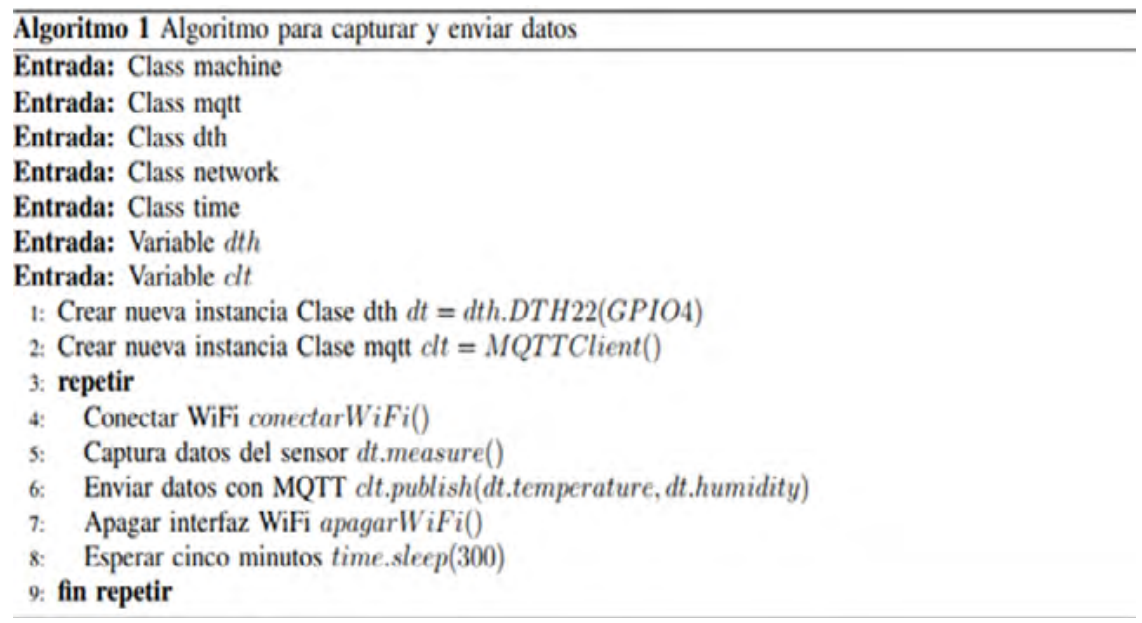

Circuito de conexión del dispositivo

El microcontrolador se alimenta de corriente continua a través de su puerto micro USB, desde una fuente de alimentación con capacidad de $8000 \mathrm{mAh}$. La fuente de alimentación es recargada mediante un panel solar integrado. El microcontrolador nodeMCU ESP8266 dispone de una interfaz de entrada/ salida de propósito general (GPIO) para la conexión con el sensor AM2302, lo realiza a través del GPIO4 (D2) y el sensor de lluvia MH-RD al GPIO13(D7). Los sensores utilizan un protocolo de comunicación serial para enviar los datos al nodeMCU. En la Fig. 2 podemos observar la interconexión de los sensores con el microcontrolador.

Figura 3. Circuito del dispositivo de monitoreo

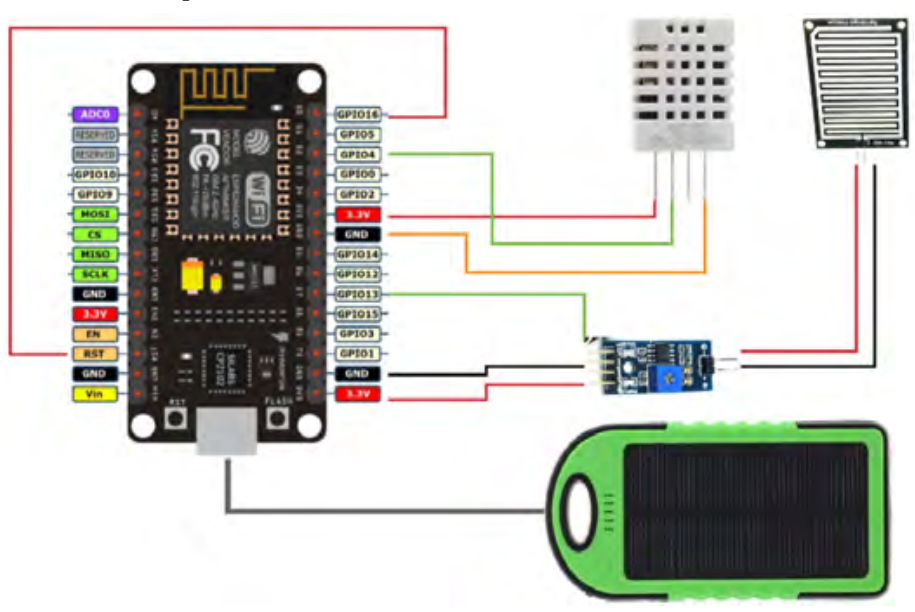

\section{Implementación de la plataforma de monitoreo}

Servidor de traducción de mensajes o Broker

El elemento encargado de recibir los datos del dispositivo de monitoreo es conocido con el nombre servidor de traducción de mensajes o bróker. En el 
presente trabajo utiliza un bróker de código abierto conocido como mosquitto, trabaja con redes de alta latencia y bajo ancho de banda (Mosquitto, 2018). En la Fig. 4 se muestra el funcionamiento del bróker al momento de recibir información de un dispositivo de monitoreo.

Figura 4. Recepción de mensajes en el bróker.

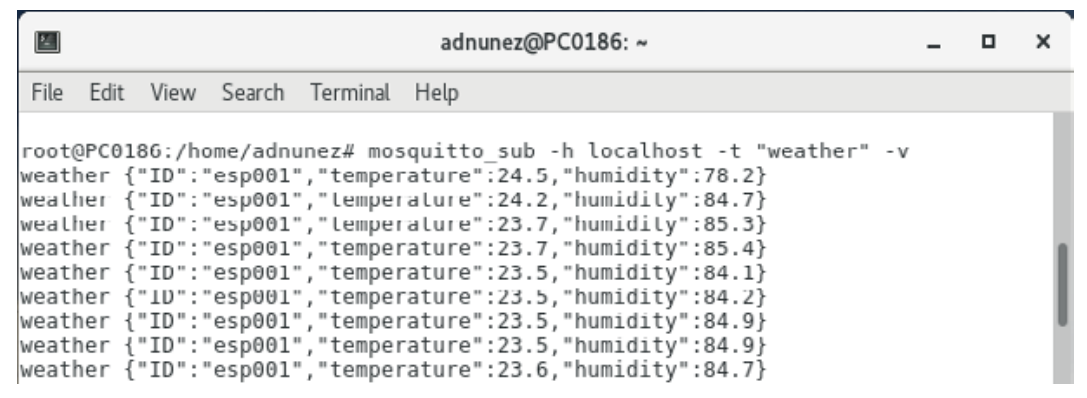

Arquitectura de la plataforma de monitoreo

Durante el diseño del software de la plataforma de monitoreo se identificaron dos elementos principales que interactúan con la plataforma y el dispositivo de medición ambiental: El primer elemento es el servidor mosquitto que se encarga de la traducción de mensajes, el cual recibe los datos enviados por el dispositivo de monitoreo a través del protocolo MQTT y los pasa a la aplicación para que sean procesados. El segundo elemento es la aplicación web que permite al usuario observar en tiempo real la temperatura, humedad y precipitación, a través de una interfaz gráfica o dashboard y además registra todos los datos en la base de datos. En la Fig. 5 se puede observar la plataforma de monitoreo basada en IoT.

Figura 5. Arquitectura de implementación de la plataforma de monitoreo.

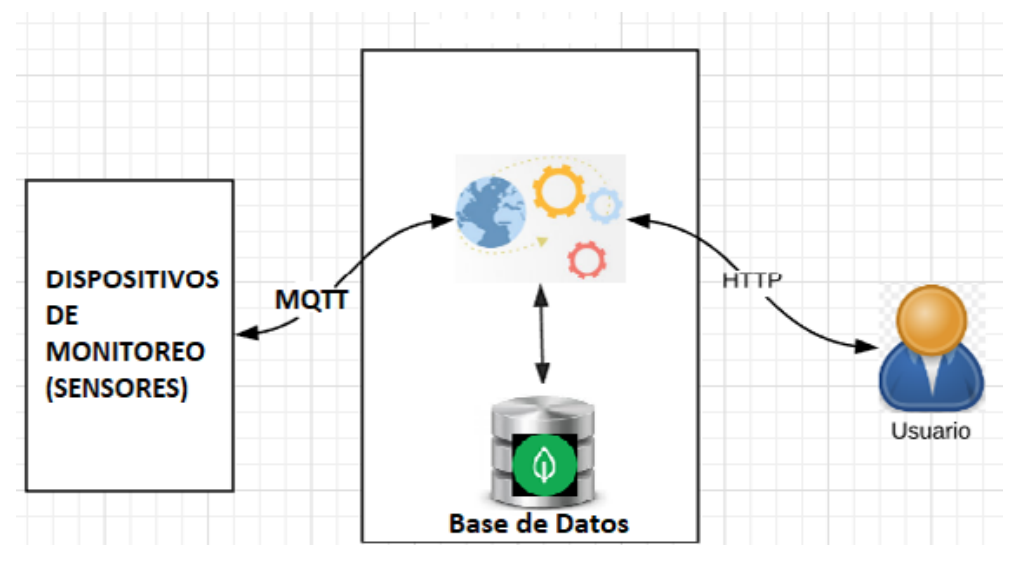

Desarrollo de la aplicación web

La aplicación web permite realizar dos funciones principales: la primera función consiste en guardar la información en la base de datos y la segunda permite mostrar esta información a través de una interfaz gráfica o dashboard. La aplicación fue desarrollada utilizando la herramienta node-red debido a 
que permite conectar: dispositivos de hardware, APIs, servicios en línea y además dispone de un editor basado en navegador, que facilita la conexión mediante flujos de datos (Node-Red, 2018). En la Fig. 6 se observa el desarrollo de la interfaz gráfica utilizando la herramienta node-red.

Figura 6. Desarrollo de la aplicación con la herramienta node-red.

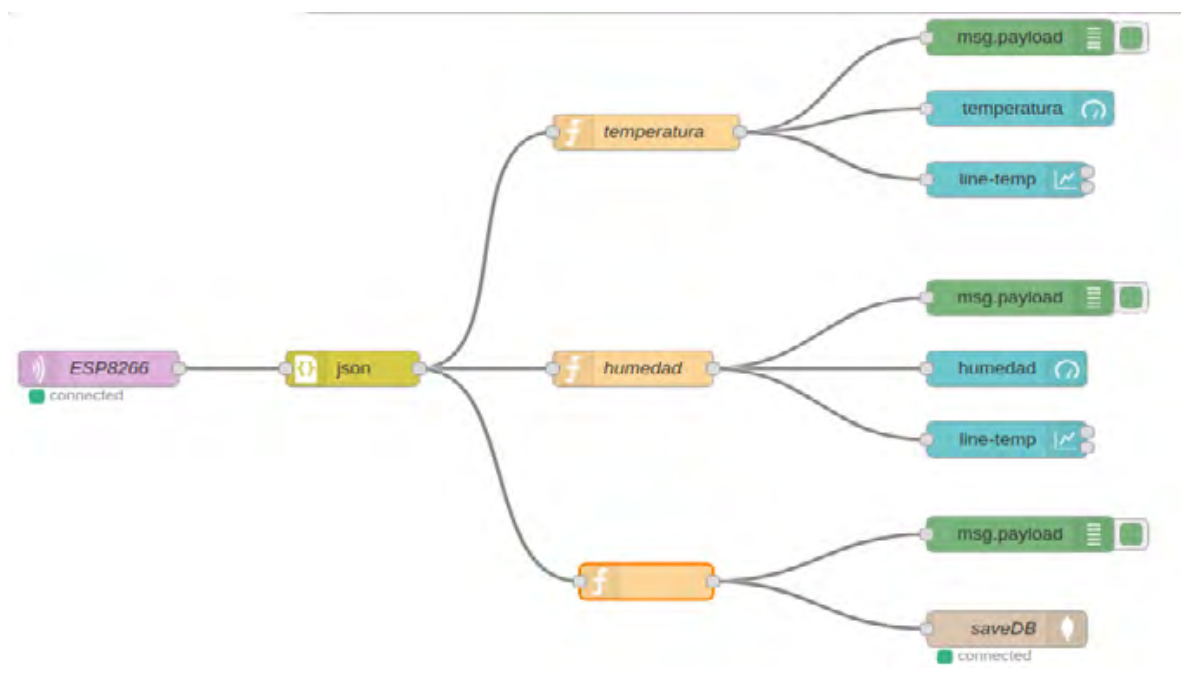

Almacenamiento de los datos

Con respecto a la permanencia de los datos se escogió la base de datos no relacional denominada mongoDB. Este tipo de base de datos permite almacenar cualquier tipo de datos, analizarlos en tiempo real y cambiar el esquema a medida que avanza la implementación de dispositivos de IoT. Estas característica son importantes cuando es necesario agregar nuevos dispositivos con distintos tipos de datos(MongoDB, 2018), de acuerdo a un análisis cuantitativo realizado por (Patil, Hanni, Tejeshwar, \& Patil, 2017).

El presente trabajo está en la fase de recopilación de datos de temperatura, humedad y de lluvia, por lo tanto, el incremento de diferentes sensores conlleva a recibir y almacenar distintos tipos de datos. Por este motivo se requiere de una base de datos que se adapte a estos cambios, como mongoDB.

\section{RESULTADOS}

El experimento fue realizado en las parcelas de la Carrera de Ingeniería Agropecuaria de la Universidad de las Fuerzas Armadas ESPE Sede Santo Domingo. Para analizar los resultados obtenidos se los ha clasificados en tres secciones: (1) Dispositivo de monitoreo ambiental, (2) Interfaz gráfica para análisis en tiempo real y (3) Validación de los datos obtenidos. 
Dispositivo de monitoreo ambiental

Como podemos observar en la Fig.7 el sensor humedad/temperatura AM2302 y el sensor de lluvia MH-RD se encuentran conectados al microcontrolador nodeMCU ESP8266. El microcontrolador se alimenta de energía eléctrica mediante un power bank a través de un cable usb.

Figura 7. Dispositivo de monitoreo

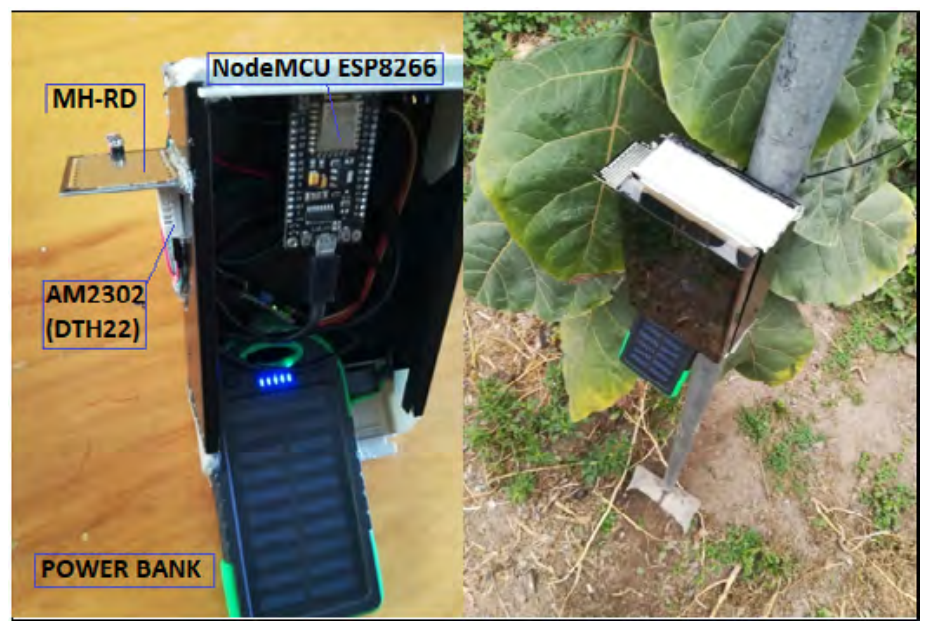

Presentación de la interfaz gráfica o dashboard

La interfaz gráfica tiene la función principal de mostrar los datos obtenidos a partir de la implementación de los diferentes dispositivos de monitoreo. En la Fig. 8 se puede observar el resultado en tiempo real de uno de los sensores.

Figura 8. Interfaz gráfica que muestra datos en tiempo real.

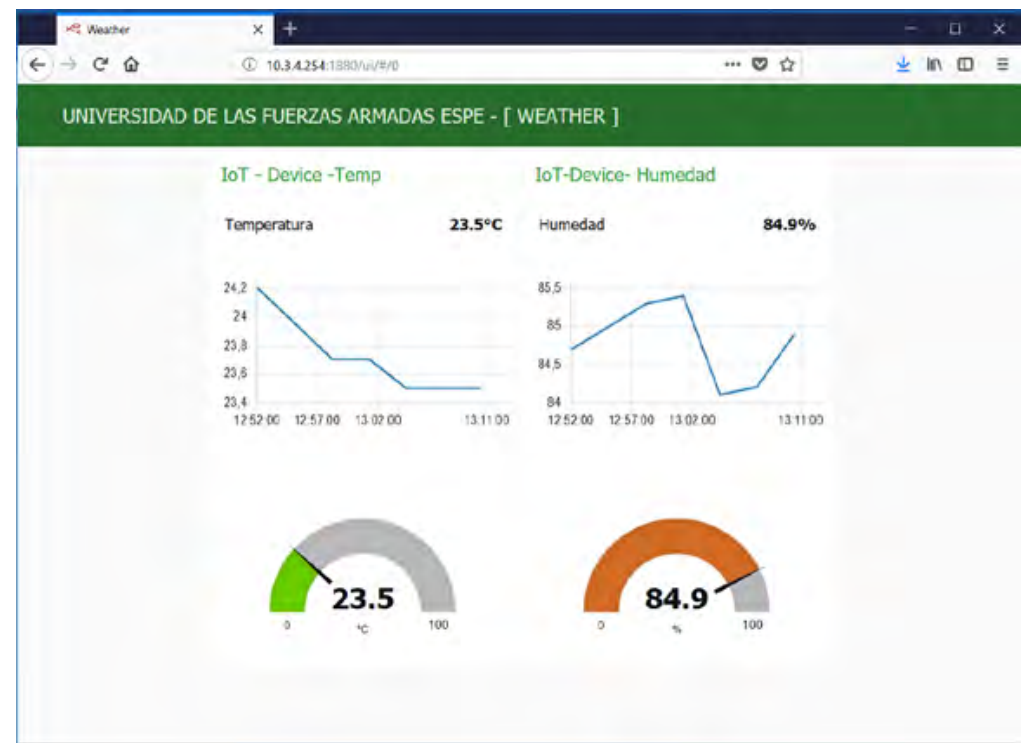

\section{Cumbres}


Validación de datos obtenidos

Para validar los datos obtenidos se realizó un análisis comparativo entre los datos entregados por tres dispositivos; primero, el dispositivo de monitoreo propuesto; segundo, un termohigrómetro modelo Fluke 971, el cual es el testigo y como tercer medio de comparación se utilizó la aplicación google weather. En la tabla 1, se muestra un extracto de las pruebas realizadas.

Tabla 1.Tabla de resultados de las mediciones de humedad y temperatura con el Dispositivo de Monitoreo, un Thermohigrometro y la aplicación Google Weather.

\begin{tabular}{|c|c|c|c|c|c|c|}
\hline \multirow[t]{2}{*}{ ORD } & \multicolumn{2}{|c|}{$\begin{array}{l}\text { DISPOSITIVO DE MONITO- } \\
\text { REO }\end{array}$} & \multicolumn{2}{|c|}{ THERMOHIGROMETRO } & \multicolumn{2}{|c|}{ GOOGLE WEATHER } \\
\hline & Temperatura & Humedad & Temperatura & Humedad & Temperatura & Humedad \\
\hline 1 & 24.9 & 86 & 24.7 & 85.8 & 24 & 80 \\
\hline 2 & 25.1 & 83.3 & 24.9 & 83.2 & 24 & 80 \\
\hline 3 & 25.4 & 81.9 & 25.2 & 82.0 & 24 & 80 \\
\hline 4 & 25.7 & 80.9 & 25.3 & 79.9 & 24 & 80 \\
\hline 5 & 26 & 80.9 & 25.9 & 79.9 & 24 & 80 \\
\hline 6 & 26 & 79.7 & 25.9 & 78.8 & 24 & 80 \\
\hline 7 & 26.2 & 81.2 & 26.0 & 79.9 & 24 & 80 \\
\hline 8 & 26.2 & 81.1 & 25.9 & 80.5 & 24 & 80 \\
\hline 9 & 26.1 & 81.3 & 25.8 & 80.8 & 24 & 80 \\
\hline 10 & 26 & 80.2 & 25.9 & 79.9 & 24 & 80 \\
\hline 11 & 26 & 80.2 & 25.8 & 80.1 & 24 & 80 \\
\hline 12 & 26.3 & 80.8 & 26.1 & 80.3 & 24 & 80 \\
\hline
\end{tabular}

Estas mediciones fueron realizadas cada 5 minutos durante 4 horas diarias en 5 días. En el caso de nuestro dispositivo, los datos se recolectaron automáticamente, usando nuestra aplicación, mientras que en el caso del termohigrómetro y google weather, se registraron de forma manual. Cabe aclarar que la aplicación google weather, solo entrega datos de temperatura cada hora, lo que lo hace menos preciso.

\section{CONCLUSIONES}

La disponibilidad de hardware de bajo costo y el acceso a herramientas de código abierto permitieron desarrollar un dispositivo de medición de variables climáticas de bajo costo y una plataforma que gestiona los datos del dispositivo basado en IoT.

El dispositivo permitió capturar datos y enviarlos a un servidor, para que sean procesados y almacenados. La interfaz gráfica desarrollada permite analizar de manera visual la información en tiempo real.

Al calcular el error medio entre nuestro dispositivo y el dispositivo testigo se pudo comprobar que la exactitud en la medición de la temperatura fue de un $96.2 \%$, mientras que la exactitud en la humedad fue de un $98.4 \%$. 
Este podría ser un punto de partida para los agricultores que están interesados en implementar este tipo de tecnología a su producción.

Como trabajo futuro, tenemos previsto establecer un modelo que permita predecir con precisión, las diferentes variables climáticas, a partir de los datos recolectados por los dispositivos de monitoreo en tiempo real.

\section{REFERENCIAS BIBLIOGRÁFICAS}

Barnaghi, P., Sheth, A., \& Henson, C. (2013). From data to actionable knowledge: Big data challenges in the web of things. IEEE Intelligent Systems, 28(6). https://doi.org/10.1109/MIS.2013.142

CISCO. (2018). Cisco visual networking index: forecast and methodlogy, 2012c2017. Retrieved from: https://www.cisco.com/c/en/us/solutions/collateral/serviceprovider/visual- networking-index-vni/white-paper-c11-741490.html

Dholu, M., \& Ghodinde, K. A. (2018). Internet of Things (IoT) for Precision Agriculture Application. Proceedings of the 2nd International Conference on Trends in Electronics and Informatics, ICOEI 2018, (Icoei), 339-342. https://doi. org/10.1109/ICOEI.2018.8553720

Guo, T., \& Zhong, W. (2015). Design and implementation of the span greenhouse agriculture Internet of Things system. Proceedings of 2015 International Conference on Fluid Power and Mechatronics, FPM 2015, 398-401.

https://doi.org/10.1109/FPM.2015.7337148

Halder, S., \& Sivakumar, G. (2018). Embedded based remote monitoring station for live streaming of temperature and humidity. International Conference on Electrical, Electronics, Communication Computer Technologies and Optimization Techniques, ICEECCOT 2017, 2018-January, 284-287.

https://doi.org/10.1109/ICEECCOT.2017.8284683

Heble, S., Kumar, A., Prasad, K. V. V. D., Samirana, S., Rajalakshmi, P., \& Desai, U. B. (2018). A low power IoT network for smart agriculture. IEEE World Forum on Internet of Things, WF-IoT 2018 - Proceedings, 2018-January, 609-614. https://doi.org/10.1109/WF-IoT.2018.8355152

kopoulos, D. (2015). Do-it-Yourself Digital Agriculture applications with semantically enhanced IoT platform. 2015 IEEE 10th International Conference on Intelligent Sensors, Sensor Networks and Information Processing, ISSNIP 2015, 7-9. https://doi.org/10.1109/ISSNIP.2015.7106951

Khattab, A., Abdelgawad, A., \& Yelmarthi, K. (2017). Design and implementation of a cloud-based IoT scheme for precision agriculture. Proceedings of the International Conference on Microelectronics, ICM, 201-204. https://doi. org/10.1109/ICM.2016.7847850

Kodali, R. K., \& Mahesh, K. S. (2016). Low cost ambient monitoring using ESP8266. In Proceedings of the 2016 2nd International Conference on Contemporary Computing and Informatics, IC3I 2016 (pp. 779-782). https://doi. org/10.1109/IC3I.2016.7918788

Kodali, R. K., \& Soratkal, S. R. (2017). MQTT based home automation system using ESP8266. IEEE Region 10 Humanitarian Technology Conference 2016,

\section{Cumbress}


R10-HTC 2016 - Proceedings.

https://doi.org/10.1109/R10-HTC.2016.7906845

Ma, J., Zhou, X., Li, S., \& Li, Z. (2011). Connecting agriculture to the internet of things through sensor networks. Proceedings - 2011 IEEE International Conferences on Internet of Things and Cyber, Physical and Social Computing, Ithings /CPSCom 2011, 184-187. https://doi.org/10.1109/iThings/CPSCom.2011.32

Mangundu, E. M., Mateus, J. N., Zodi, G. A. L., \& Johson, J. (2018). A wireless sensor network for rainfall monitoring, using cellular network: A case for Namibia. 2017 Global Wireless Summit, GWS 2017, 2018-January, 240-244. https://doi.org/10.1109/GWS.2017.8300469

Mat, I., Kassim, M. R. M., \& Harun, A. N. (2014). Precision irrigation performance measurement using wireless sensor network. International Conference on Ubiquitous and Future Networks, ICUFN, 154-157. https://doi. org/10.1109/ICUFN.2014.6876771

Math, R. K., \& Dharwadkar, N. V. (2017). A wireless sensor network based low cost and energy efficient frame work for precision agriculture. 2017 International Conference on Nascent Technologies in Engineering, ICNTE 2017 Proceedings. https://doi.org/10.1109/ICNTE.2017.7947883

Math, R. K. M., \& Dharwadkar, N. V. (2019). IoT Based low-cost weather station and monitoring system for precision agriculture in India. Proceedings of the International Conference on I-SMAC (IoT in Social, Mobile, Analytics and Cloud), I-SMAC 2018, 81-86.

https://doi.org/10.1109/I-SMAC.2018.8653749

Munandar, A., Fakhrurroja, H., Rizqyawan, M. I., Pratama, R. P., Wibowo, J. W., \& Anto, I. A. F. (2018). Design of real-time weather monitoring system based on mobile application using automatic weather station. Proceedings of the 2nd International Conference on Automation, Cognitive Science, Optics, Micro Electro-Mechanical System, and Information Technology, ICACOMIT 2017, 44-47. https://doi.org/10.1109/ICACOMIT.2017.8253384

MongoDB. (2018). The MongoDB 3.6 Manual - MongoDB Manual 3.6. Retrieved from: https://docs.mongodb.com/manual/

Montoya, E. A. Q., Colorado, S. F. J., Muñoz, W. Y. C., \& Golondrino, G. E. C. (2017). Propuesta de una Arquitectura para Agricultura de Precisión Soportada en IoT. RISTI - Revista Iberica de Sistemas e Tecnologias de Informacao, (24), 39-56. https://doi.org/10.17013/risti.24.39-56

Mosquitto. (2018). Eclipse Mosquitto. Retrieved November 3, 2018, from https://mosquitto.org/

Nageswara Rao, R., \& Sridhar, B. (2018). IoT based smart crop-field monitoring and automation irrigation system. Proceedings of the 2nd International Conference on Inventive Systems and Control, ICISC 2018, (Icisc), 478-483. https://doi.org/10.1109/ICISC.2018.8399118

Node-Red. (2018). Node-RED. Retrieved October 21, 2018, from https://nodered.org/ Openhacks. (2016). RAIN SENSOR MODULE. Retrieved from https://www.openhacks.com/uploadsproductos/rain_sensor_module.pdf

Patil, M. M., Hanni, A., Tejeshwar, C. H., \& Patil, P. (2017). A qualitative 
analysis of the performance of MongoDB vs MySQL database based on insertion and retriewal operations using a web/android application to explore load balancing-Sharding in MongoDB and its advantages. Proceedings of the International Conference on IoT in Social, Mobile, Analytics and Cloud, I-SMAC 2017, 325-330.

https://doi.org/10.1109/I-SMAC.2017.8058365

Qiu, T., Xiao, H., \& Zhou, P. (2013). Frameworl and case studies of intelligence monitoring platform in facility agriculture ecosystem. 2013 2nd International Conference on Agro-Geoinformatics: Information for Sustainable Agriculture, Agro-Geoinformatics 2013, 522-525.

https://doi.org/10.1109/Argo-Geoinformatics.2013.6621976

Saini, H., Thakur, A., Ahuja, S., Sabharwal, N., \& Kumar, N. (2016). Arduino based automatic wireless weather station with remote graphical application and alerts. 3rd International Conference on Signal Processing and Integrated Networks, SPIN 2016, 605-609.

https://doi.org/10.1109/SPIN.2016.7566768

Shaout, A., Li, Y., Zhou, M., \& Awad, S. (2015). Low cost embedded weather station with intelligent system. 2014 10th International Computer Engineering Conference: Today Information Society What's Next?, ICENCO 2014, 100-

106. https://doi.org/10.1109/ICENCO.2014.7050439

Siano, P. (2014). Demand response and smart grids - A survey. Renewable and Sustainable Energy Reviews, 30, 461-478. https://doi.org/10.1016/j. rser.2013.10.022

Saputra, L. K. P., \& Lukito, Y. (2017). Implementation of air conditioning control system using REST protocol based on NodeMCU ESP8266. 2017 International Conference on Smart Cities, Automation \& Intelligent Computing Systems (ICON-SONICS), 126-130. https://doi.org/10.1109/ICON-SONICS.2017.8267834

Viani, F., Bertolli, M., Salucci, M., \& Polo, A. (2017). Low-Cost Wireless Monitoring and Decision Support for Water Saving in Agriculture. IEEE Sensors Journal, 17(13), 4299-4309. https://doi.org/10.1109/JSEN.2017.2705043

Zanella, a, Bui, N., Castellani, a, Vangelista, L., \& Zorzi, M. (2014). Internet of Things for Smart Cities. IEEE Internet of Things Journal, 1(1), 22-32. https://doi.org/10.1109/JIOT.2014.2306328

Zhang, Peng \& Zhang, Junjie \& Chen, M. (2016). Economic Impacts of Climate Change on Agriculture: The Importance of Additional Climatic Variables Other than Temperature and Precipitation. Journal of Environmental Economics and Management, 8-31. 